\title{
Sterile signals generate weaker and delayed macrophage NLRP3 inflammasome responses relative to microbial signals
}

\author{
Jelena S Bezbradica ${ }^{1}$, Rebecca C Coll ${ }^{1}$ and Kate Schroder
}

Inflammation is the host response to microbial infection or sterile injury that aims to eliminate the insult, repair the tissue and restore homeostasis. Macrophages and the NLRP3 inflammasome are key sentinels for both types of insult. Although it is well established that the NLRP3 inflammasome is activated by microbial products and molecules released during sterile injury, it is unclear whether the responses elicited by these different types of signals are distinct. In this study, we used lipopolysaccharide and tumor necrosis factor as prototypical microbial and sterile signal 1 stimuli, respectively, to prime the NLRP3 inflammasome. We then used the bacterial toxin nigericin and a common product released from necrotic cells, ATP, as prototypical microbial and sterile signal 2 stimuli, respectively, to trigger the assembly of the NLRP3 inflammasome complex in mouse and human macrophages. We found that NLRP3 inflammasome responses were weakest when both signal 1 and signal 2 were sterile, but responses were faster and stronger when at least one of the two signals was microbial. Ultimately, the most rapid and potent responses were elicited when both signals were microbial. Together, these data suggest that microbial versus sterile signals are distinct, both kinetically and in magnitude, in their ability to generate inflammasome-dependent responses. This hierarchy of NLRP3 responses to sterile versus microbial stimuli likely reflects the urgent need for the immune system to respond rapidly to the presence of infection to halt pathogen dissemination.

Cellular \& Molecular Immunology (2017) 14, 118-126; doi:10.1038/cmi.2016.11; published online 21 March 2016

Keywords: inflammasome; NLRP3; sterile inflammation; TNF

\section{INTRODUCTION}

Inflammation is broadly defined as a local response to infection or injury that aims to restore homeostasis. ${ }^{1}$ The purpose of the inflammatory response is distinct in these two situations. In infection, inflammation primarily aims to eradicate the microbe and induce protective immunity, whereas inflammation triggered by sterile injury aims to limit the damage and facilitate tissue repair. Beyond a few examples (for example, induction of specific antimicrobial or tissue repair genes), the signaling mechanisms and inflammatory mediators that discriminate these two different types of inflammatory responses, and their consequences for immune function, are poorly understood. Macrophages and their pattern recognition receptors are key cellular and molecular sentinels for both types of immune insult.

One such pattern recognition receptor, the NOD-like receptor NLRP3, senses both pathogen- and host-derived danger signals. ${ }^{2}$ NLRP3 function can be activated by a diverse range of live microbes (for example, influenza virus and bacteria such as Staphylococcus aureus) and microbial products $^{3}$ (for example, the bacterial toxin and potassium ionophore, nigericin), as well as an array of host-derived molecules released or generated during sterile injury, such as extracellular ATP released from damaged tissues and cholesterol crystals that deposit in atherosclerotic plaques. ${ }^{4}$ How exactly all these different stimuli activate NLRP3 (presumably via a common downstream mediator ${ }^{5}$ ) is an area of extensive research elsewhere. Regardless, upon activation, NLRP3 oligomerizes, triggering the assembly of a multimolecular signaling platform composed of NLRP3, the adapter ASC and the zymogen pro-caspase-1. Clustering of caspase-1 within the complex induces its activation, self-cleavage and its ability to process substrates such as interleukin (IL)- $1 \beta$. The responding

Institute for Molecular Bioscience (IMB), IMB Centre for Inflammation and Disease Research and Australian Infectious Diseases Research Centre, The University of Queensland, Brisbane, St Lucia, Queensland 4072, Australia

${ }^{1}$ These authors contributed equally to this work.

Correspondence: Dr K Schroder, Institute for Molecular Bioscience, University of Queensland, St Lucia, Queensland 4072, Australia.

E-mail: K.Schroder@imb.uq.edu.au

Received: 30 November 2015; Revised: 1 February 2016; Accepted: 1 February 2016 
cell then often dies through caspase-1-directed osmotic lysis ('pyroptosis'), which passively releases cytoplasmic proteins (alarmins) to alert neighboring cells. ${ }^{2,3}$ Caspase-1 activation and cytokine release initiate an inflammatory response to recruit further phagocytes to the site of insult, and to activate the recruited cells to destroy invading microbes and remove dead cells and debris. In the case of tissue injury, additional factors are likely to be released to coordinate the repair of damaged tissue; the identity of such factors remains currently unknown. Inflammation wanes with the clearance of the insult, until homeostasis is restored. If however NLRP3 activation is prolonged or inappropriate, inflammatory disease can develop.

NLRP3 function is generally beneficial in antimicrobial defense, but pathological in several inherited autoinflammatory diseases and acquired inflammatory diseases. For example, NLRP3 is critically required for antifungal defense against Candida albicans ${ }^{6}$ and Paracoccidioides brasiliensis ${ }^{7}$ in vivo. NLRP3 is also involved in the recognition of numerous bacterial pathogens, ${ }^{8}$ including gram-negative species such as Salmonella typhimurium ${ }^{9}$ and Burkholderia pseudomallei. ${ }^{10}$ Conversely, gain-of-function NLRP3 mutations drive inherited autoinflammatory diseases that are characterized by multi-organ inflammatory episodes, fevers and neutrophilia, collectively termed Cryopyrin-associated periodic fever syndromes. ${ }^{11}$ In acquired inflammatory diseases, aberrant production of hostderived molecules trigger NLRP3-dependent pathology. For example, NLRP3 is activated by monosodium urate crystals, ${ }^{12}$ islet amyloid polypeptide ${ }^{13}$ and fibrillar amyloid-beta, ${ }^{14,15}$ in gout, type 2 diabetes and Alzheimer's disease, respectively. The resultant production of IL- $1 \beta$, and induction of cell lysis and consequent alarmin release, then drives pathological inflammation. Thus, in many such diseases, pharmacological blockade of IL- $1 \beta$ alleviates disease-associated pathology and genetic deletion of Nlrp3 is protective. ${ }^{12,15}$

In vitro NLRP3 function requires primary myeloid cells to receive two distinct signals in succession. Signal 1 is a 'priming' signal that upregulates NLRP3 expression and also induces the expression of pro-IL-1 $\beta$, which is not expressed in resting myeloid cells. Microbial molecules (for example, bacterial lipopolysaccharide (LPS)) or host cytokines (for example, tumor necrosis factor (TNF), which is locally released at sites of tissue damage $)^{16,17}$ can function as signal 1 in this context, but most experimental protocols use LPS as a priming signal. Signal 2 is an activating signal and triggers the assembly of the NLRP3 inflammasome. Similar to signal 1, signal 2 can be derived from either the microbe (for example, the bacterial toxin, nigericin) or the host (for example, extracellular ATP). Whole microbes themselves often provide both signal 1 and signal 2, and likewise, tissue damage often generates the concurrent release of cytokines and alarmins that can serve as sterile signals 1 and $2 .{ }^{18}$

A common feature of inflammasome-priming signals is that they activate the NF- $\mathrm{KB}$ pathway to upregulate NLRP3 and pro-IL-1 $\beta$ expression. ${ }^{17}$ Consistent with this, mice deficient in the negative regulator of NF- $\mathrm{KB}, \mathrm{A} 20$, exhibit overactive NLRP3 signaling and develop NLRP3-dependent inflammatory diseases (for example, arthritis ${ }^{19,20}$ ). Toll-like receptor and C-type lectin receptor agonists activate NF- $\mathrm{KB}$ and prime the NLRP3 inflammasome. 6,17,21,22 Among the signals associated with sterile inflammation (for example, RANKL, FasL), many can activate the NF- $\mathrm{\kappa B}$ pathway, but only some (for example, TNF, IL-1 $\alpha$, IL-1 $\beta$ ) appear able to prime the NLRP3 inflammasome. ${ }^{16,23,24}$ Of these, TNF appears to be the most potent priming stimulus for myeloid cell NLRP3 responses, and TNF is chronically elevated in many sterile inflammatory diseases whose pathology is worsened by uncontrolled NLRP3 inflammasome activation (for example, arthritis ${ }^{25}$ and metabolic syndrome ${ }^{26}$ ).

Although the ability of sterile injury signals (for example, extracellular ATP, necrotic cells and hyaluronan) to activate the NLRP3 inflammasome is well established, surprisingly, a direct side-by-side comparison of NLRP3 inflammasome responses to microbial versus sterile priming and activating signals is not yet reported. In fact, very few reports have studied NLRP3 responses in a fully sterile activation model, in which both priming and activating stimuli are host derived. In this study, we sought to understand whether sterile versus microbial NLRP3 priming and activating signals trigger similar or distinct macrophage inflammasome-dependent responses, as such information may be important for the design of new drugs to treat inflammatory diseases. We found that the sterile priming signal TNF exhibited weaker and delayed ability to prime for NLRP3 responses as compared to the microbial priming signal, LPS. Likewise, the sterile NLRP3 agonist, ATP, was a less potent activator of macrophage NLRP3 responses than the microbial NLRP3 agonist, nigericin, in both mouse and human macrophages. Thus, sterile signals generate tempered NLRP3 inflammasome responses relative to microbial signals.

\section{MATERIALS AND METHODS}

Mouse and human primary macrophage cell culture C57BL/6 and Ice ${ }^{-/-27}$ mice were housed in specific pathogenfree facilities at the University of Queensland. Ice ${ }^{-1-}$ mice were backcrossed at least 10 times to C57BL/6. Buffy coats from healthy human donors were obtained from the Australian Red Cross Blood Service. Peripheral blood mononuclear cells were isolated by density centrifugation using Ficoll-Paque Plus (GE Healthcare, Parramatta, NSW, Australia). CD14+ monocytes were then isolated using magnetic-activated cell sorting CD14+ positive selection (Miltenyi Biotech, Macquarie Park, NSW, Australia), according to the manufacturer's instructions. Human and murine macrophages were differentiated from human $\mathrm{CD} 14+$ monocytes and murine bone marrow as previously described. ${ }^{28}$ The University of Queensland Animal Ethics Committee approved all experimental protocols involving mice, and all studies using primary human cells were approved by the University of Queensland Human Medical Research Ethics Committee.

Murine bone marrow-derived macrophages (BMMs) and human monocyte-derived macrophages (HMDMs) were cultured in RPM1 1640 medium (Life Technologies, Mulgrave, VIC, Australia) supplemented with $10 \%$ heat inactivated fetal 
calf serum, $2 \mathrm{~mm}$ GlutaMAX (Life Technologies), $50 \mathrm{U} / \mathrm{ml}$ penicillin-streptomycin (Life Technologies) and $150 \mathrm{ng} / \mathrm{ml}$ recombinant human macrophage colony-stimulating factor (endotoxin free, expressed and purified by the University of Queensland Protein Expression Facility).

\section{Inflammasome and pyroptosis assays}

BMMs were seeded at $1 \times 10^{6}$ cells/ml and HMDMs at $0.7 \times 10^{6}$ cells $/ \mathrm{ml}$ in 96 -well plates. Cells were stimulated with $100 \mathrm{ng} / \mathrm{ml}$ ultrapure Escherichia coli K12 LPS (Invivogen, San Diego, CA, USA), $100 \mathrm{ng} / \mathrm{ml}$ endotoxin-free recombinant murine or human TNF- $\alpha$ (Peprotech, Rocky Hill, NJ, USA), $1.25 \mathrm{~mm}$ ATP (Sigma-Aldrich, Sydney, NSW, Australia) and/or $5 \mu \mathrm{M}$ nigericin (Sigma-Aldrich) as indicated. IL-1 $\beta$ levels in cellfree supernatants were analyzed by enzyme-linked immunosorbent assay (ELISA; eBioscience, San Diego, CA, USA). Cytotoxicity was analyzed by lactate dehydrogenase (LDH) release (CytoTox 96 Promega, Alexandria, NSW, Australia).

\section{Western blotting}

Cell lysates (extracts) were prepared by lysis in boiling buffer (66 mm Tris- $\mathrm{Cl} \mathrm{pH} \mathrm{7.4,} \mathrm{2 \%} \mathrm{sodium} \mathrm{dodecyl} \mathrm{sulfate} \mathrm{(SDS)).} \mathrm{Cell}$ lysates (extracts) and supernatants were subjected to SDS-PAGE, and transferred to nitrocellulose membranes. Primary antibodies used were caspase-1 (Casper-1, Adipogen, San Diego, CA, USA, at 1:1000), NLRP3 (AG-20B-0014, Cryo2, Adipogen at 1:1000), I $\mathrm{KB} \alpha$ (4812, clone 44D4, Cell Signaling Technology, Danvers, MA, USA, at 1:1000), IL-1 $\beta$ (AF-401NA, R\&D Systems, Minneapolis, MN, USA, at 1:2000), Tubulin (T5168, clone B-5-1-2, Sigma-Aldrich at 1:2000) and GAPDH (2275-PC, R\&D Systems at 1:5000).

\section{Statistical analysis}

Two-tailed students $t$-tests (paired for human data and unpaired for mouse) were performed using Prism GraphPad software (La Jolla, CA, USA). Data were considered significant when $\left.P \leqslant 0.05\left(^{*}\right), 0.005^{* *}\right), 0.001\left(^{* * *}\right)$ or $\left.0.0001^{* * *}\right)$.

\section{RESULTS}

Sterile signals 1 and 2 generate weak and delayed NLRP3 inflammasome responses in mouse macrophages

To test whether NLRP3 inflammasome responses to microbial versus sterile signals are distinct, we stimulated wild-type (WT) murine BMMs with a microbial (LPS) or sterile (TNF) signal 1 for 24 or $6 \mathrm{~h}$ before, or at the same time as $(0 \mathrm{~h}$, 'co-treatment') signal 2 to mimic chronic versus acute inflammasome priming. The signal 2 used to trigger NLRP3 activation was either microbial (nigericin) or sterile (ATP), and every possible combination of sterile versus microbial signals 1 and 2 were assayed for response (Figure 1a). Inflammasome activation was monitored $3 \mathrm{~h}$ later using well-established experimental readouts. LDH release assay monitored caspase-1-dependent cell death, ELISAs quantified caspase-1-dependent IL-1 $\beta$ secretion, and immunoblotting examined pro-caspase-1 (p46) cleavage and the release of caspase-1 p20 into the culture supernatant. Caspase-1-deficient $\left(\mathrm{Ice}^{-/-}\right)$macrophages were assayed in parallel to control for the inflammasome dependency of each response. We observed that acute LPS exposure (co-treatment with signal 2 at $0 \mathrm{~h}$ ) efficiently primed for inflammasome responses by nigericin and ATP, as measured by cell death and caspase-1 cleavage; pro-IL-1 $\beta$ expression was not induced at this early time and so as anticipated, IL-1 $\beta$ was not secreted under any co-treatment conditions (Figures $1 \mathrm{~b}-\mathrm{d}$ ). By contrast to LPS, when TNF was co-administered with ATP or nigericin, it only weakly primed for caspase-1 cleavage and pyroptosis (Figures $1 \mathrm{~b}-\mathrm{d}$ ). When present chronically ( 6 or $24 \mathrm{~h}$ ), both LPS and TNF could prime for NLRP3 responses, but the microbial signal 1 (LPS) primed for cell death, IL-1 $\beta$ secretion and caspase- 1 cleavage with much faster and more transient kinetics (peak response at $6 \mathrm{~h}$, Figures $1 \mathrm{e}-\mathrm{j}$ ) compared with sterile priming with TNF (persistent response between 6 and $24 \mathrm{~h}$, Figures $1 \mathrm{e}-\mathrm{j}$ ). Together, these data broadly agree and expand on a previous study, ${ }^{16}$ and suggest that microbial versus sterile signal 1 stimuli are distinct, at least kinetically, in their ability to prime inflammasome-dependent responses.

The magnitude of responses to microbial versus sterile signals 1 and 2 also showed a hierarchy. With 0 and $6 \mathrm{~h}$ priming, cell death, IL-1 $\beta$ secretion and caspase- 1 cleavage were much more robust in response to microbial (LPS) than to sterile (TNF) signal 1. Only after $24 \mathrm{~h}$ when cells were no longer responsive to microbial (LPS) signal 1, the response to sterile (TNF) priming became dominant (Figures $1 \mathrm{~b}-\mathrm{j}$ ). Consistently, cell death, IL-1 $\beta$ secretion and caspase- 1 cleavage were always more robust in response to microbial (nigericin) than to sterile (ATP) signal 2 (Figures $1 \mathrm{~b}-\mathrm{j}$ ) regardless of priming time or the nature of the signal 1 . The combination of sterile signals 1 and 2 (TNF+ATP) was the weakest of all the combinations for 0 and $6 \mathrm{~h}$ priming time. For $24 \mathrm{~h}$ priming (at which time LPS no longer effectively primes), the cell death and caspase-1 cleavage responses to sterile signals (TNF+ATP) were weaker than their counterpart responses to TNF+nigericin. For all experiments, ATP and nigericin concentrations were titrated for maximal NLRP3 responses (Figures $2 \mathrm{a}-\mathrm{d}$ ), and the most effective dose was used to elicit NLRP3 responses. Together, these data suggest that microbial signal 1 and 2 stimuli generate heightened NLRP3 inflammasome responses as compared to sterile signal 1 and 2 stimuli.

\section{Poor responses to sterile signals cannot be overcome by} increasing the concentration of the priming stimulus

To test whether weak WT BMM responses to sterile signal 1 (TNF) are due to insufficient concentrations of this cytokine, we applied escalating doses up to $800 \mathrm{ng} / \mathrm{ml}$ for $6 \mathrm{~h}$, an optimal time at which BMM are responsive to both microbial and sterile signal 1 stimuli (Figure 1). BMM were also primed with microbial signal 1 (LPS at the supra-maximal dose of $100 \mathrm{ng} /$ $\mathrm{ml}$ ) for $6 \mathrm{~h}$ in parallel. Macrophages were then exposed to microbial (nigericin) or sterile (ATP) signal 2, and inflammasome-induced cell death and caspase-1 cleavage were monitored after $3 \mathrm{~h}$. Increased concentrations of TNF did not improve inflammasome responses, and the microbial (LPS) signal 1 remained superior to sterile (TNF) signal 1 in priming 
a

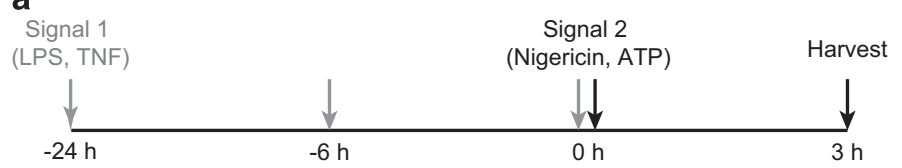

b

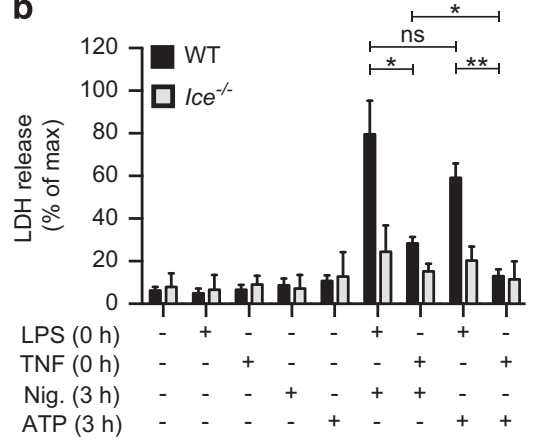

e

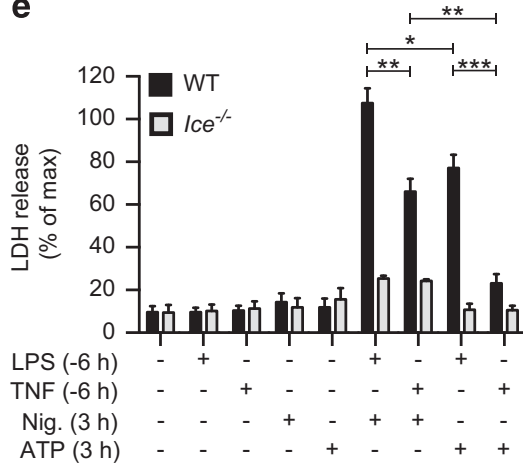

h

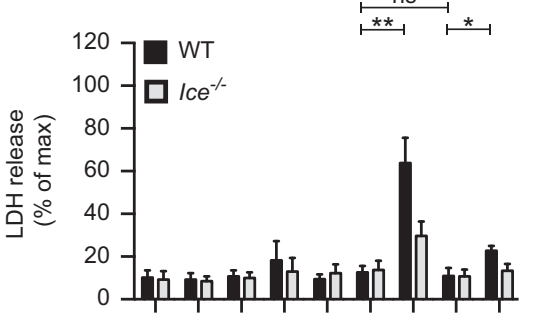

C

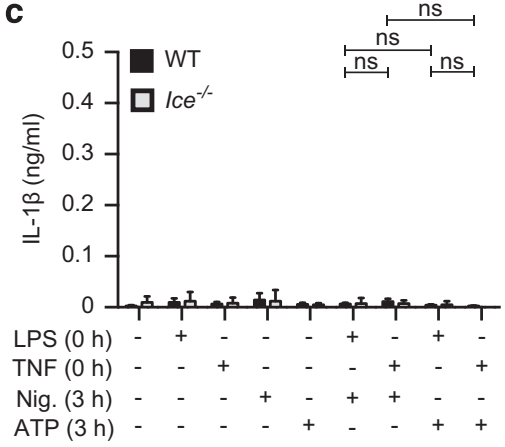

f$$
\text { A }
$$

i

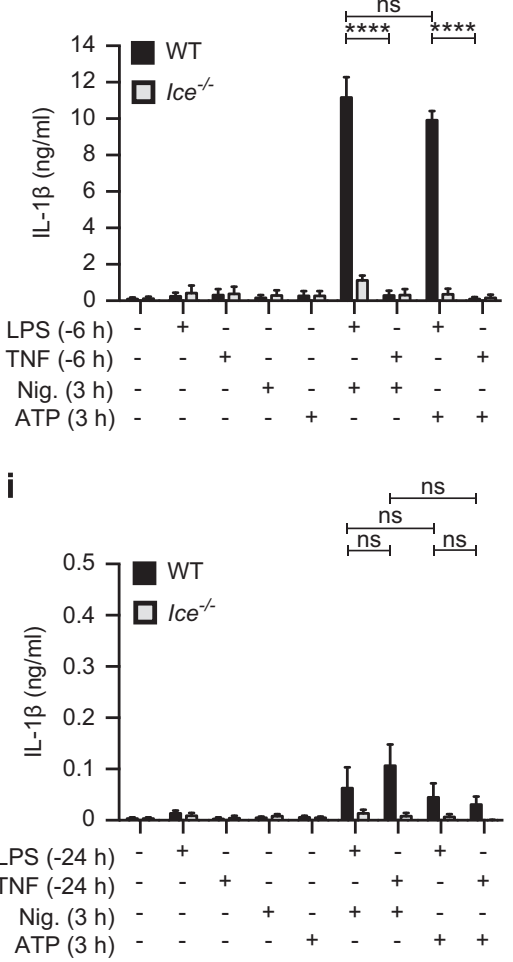

d

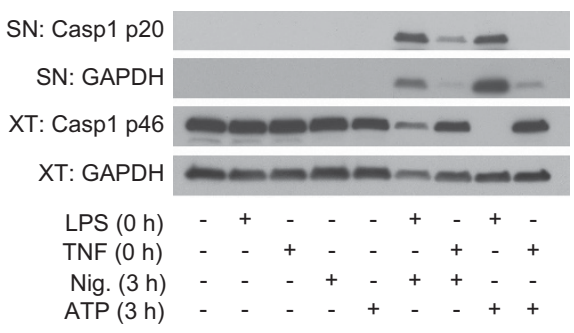

g

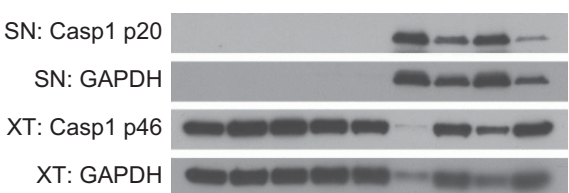

$\operatorname{LPS}(-6 \mathrm{~h}) \quad-\quad+\quad-\quad+-+$

$\operatorname{TNF}(-6 \mathrm{~h}) \quad-\quad+\quad-\quad-\quad+\quad+$

Nig. $(3 \mathrm{~h})-\mathrm{C}_{-}+-++-$

ATP $(3 \mathrm{~h})-{ }_{-}-{ }_{-}+-+$ j

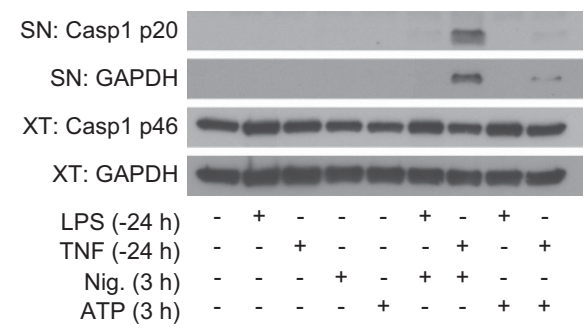

Figure 1 Sterile signals generate weak and delayed inflammasome responses in mouse macrophages. (a) Experimental outline for data $\mathbf{b}-\mathbf{j}$ : WT and $/ \mathrm{ce}^{-/-}$BMMs were left untreated or stimulated with signal 1 (100 ng/ml LPS or $100 \mathrm{ng} / \mathrm{ml}$ TNF) for 24 , 4-6 or $0 \mathrm{~h}$ (that is, signal 1 added immediately before signal 2), followed by signal 2 (1.25 mm ATP or $5 \mu \mathrm{m}$ nigericin) for $3 \mathrm{~h}$. XTs and cell-free SNs were collected. Cell death was assessed by LDH assay, and ELISA measured IL-1 $\beta$ release. Caspase- 1 levels in XT and release of cleaved p20 caspase-1 fragment into the SN were measured by immunoblot. GAPDH was used as a loading control. BMMs were stimulated with signal 1 for (b-d) $1 \mathrm{~min},(\mathbf{e}-\mathbf{g}) 4-6 \mathrm{~h}$ or $(\mathbf{h}-\mathbf{j}) 24 \mathrm{~h}$ before signal 2 . Histograms are mean+s.e.m. of data pooled from $n=3(\mathbf{b}, \mathbf{c})$ or $n=4(\mathbf{e}, \mathbf{f}, \mathbf{h}, \mathbf{i})$ independent experiments. Representative immunoblots of $n=3$ (d) and $n=4(\mathbf{g}, \mathbf{j})$ independent experiments are shown. BMM, bone marrow-derived macrophages; ELISA, enzyme-linked immunosorbent assay; IL, interleukin; LDH, lactate dehydrogenase; LPS, lipopolysaccharide; SN, supernatant; TNF, tumor necrosis factor; WT, wild type; XT, cell extract.

inflammasome responses to either microbial (nigericin, Figure 2e) or sterile (ATP, Figure 2f) signal 2 stimuli. This indicates that the poor inflammasome responses generated by sterile signals (TNF and ATP) were not due to insufficient concentration of the priming stimulus.
TNF induces weaker but more sustained NLRP3 and

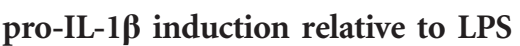

As efficient NLRP3 inflammasome assembly requires upregulated NLRP3 protein expression, ${ }^{17}$ and IL-1 $\beta$ maturation requires the induction of pro-IL-1 $\beta$ expression, we next 

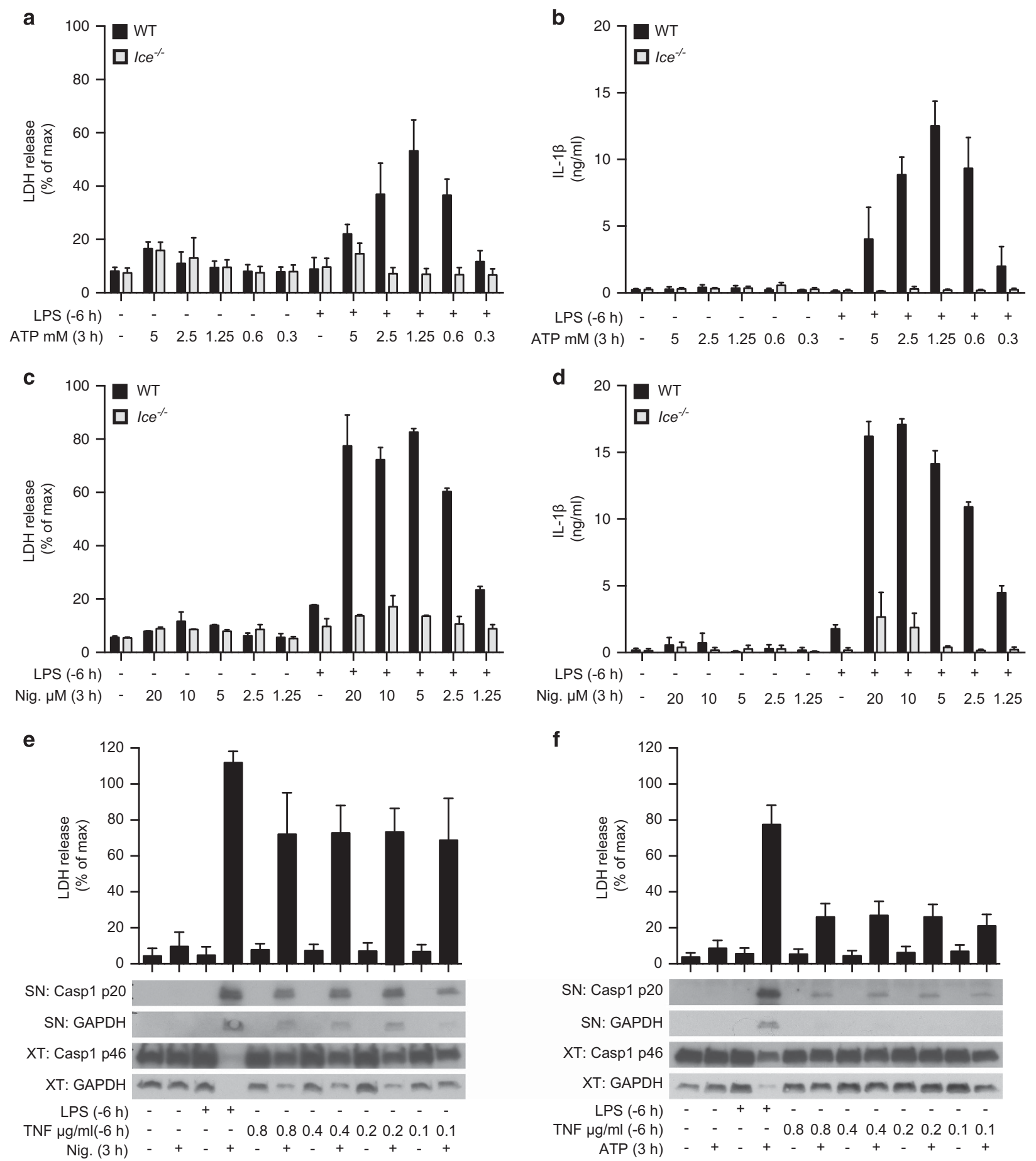

Figure 2 Poor responses to sterile signals are not due to insufficient priming stimulus dose. (a-d) WT and $/ c e^{-/-}$BMMs were left untreated ('-') or were stimulated with $100 \mathrm{ng} / \mathrm{ml}$ LPS for $6 \mathrm{~h}$ followed by escalating doses of ATP (5-0.3 mm in a, b) or nigericin (20-1.25 $\mu \mathrm{M}$ in c, d) for $3 \mathrm{~h}$. Cell death was assessed by LDH release assay $(\mathbf{a}, \mathbf{c})$ and IL-1 $\beta$ release was measured by ELISA (b, d). Histograms are mean+s. e.m. of data pooled from $n=4(\mathbf{a}, \mathbf{b})$ and mean+range from $n=2(\mathbf{c}, \mathbf{d})$ independent experiments. (e, f) WT and $I c e^{-l-}$ BMMs were left untreated ('-') or were stimulated with $100 \mathrm{ng} / \mathrm{ml}$ LPS or $800-100 \mathrm{ng} / \mathrm{ml}$ TNF for $6 \mathrm{~h}$ followed by $5 \mu \mathrm{M}$ nigericin (e), or $1.25 \mathrm{~mm}$ ATP (f), for $3 \mathrm{~h}$. Cell death was assessed by LDH release assay (upper), and caspase-1 cleavage was measured by immunoblot, relative to a GAPDH loading control (lower). Histograms are mean+s.e.m. of data pooled from $n=3$ independent experiments. Immunoblots representative of $n=3$ independent experiments are shown. BMM, bone marrow-derived macrophages; ELISA, enzyme-linked immunosorbent assay; IL, interleukin; LDH, lactate dehydrogenase; LPS, lipopolysaccharide; TNF, tumor necrosis factor; WT, wild type. 
examined NLRP3 and pro-IL- $1 \beta$ regulation by microbial (LPS) versus sterile (TNF) priming signals over a $24 \mathrm{~h}$ time course (Figures $3 \mathrm{a}$ and $\mathrm{b}$ ). Microbial signal 1 (LPS) induced NLRP3 and pro-IL-1 $\beta$ protein expression within $2 \mathrm{~h}$, which further increased until they peaked at $4 \mathrm{~h}$ (pro-IL-1 $\beta$ ) and $5 \mathrm{~h}$ (NLRP3) poststimulation. By $24 \mathrm{~h}$ post-LPS, NLRP3 and pro-IL-1 $\beta$ expression had declined to near that of unstimulated cells, likely due to LPS-induced IL-10 that suppresses NLRP3 and pro-IL-1 $\beta$ expression. ${ }^{29}$ Sterile signal 1 (TNF) also induced NLRP3 and pro-IL-1 $\beta$ expression within $2 \mathrm{~h}$ of stimulation, with NLRP3 expression peaking around $4-5 \mathrm{~h}$ post-TNF. However, TNF-induced NLRP3, and particularly TNFinduced pro-IL- $1 \beta$, expression levels were considerably lower than those induced by LPS. Importantly, NLRP3 and pro-IL-1 $\beta$ levels induced by TNF remained relatively constant, and did not decline even after $24 \mathrm{~h}$ of TNF treatment, in contrast to their LPS induction profiles. Thus, the peak expression levels of NLRP3 and pro-IL-1 $\beta$ induced by LPS (high at $2-6 \mathrm{~h}$ ) and TNF (moderately high for NLRP3 and low for pro-IL-1 $\beta$ at $2-24 \mathrm{~h}$ ) mirror the extent of inflammasome responses seen with these priming signals in Figure 1.

Weaker induction of NLRP 3 and pro-IL- $1 \beta$ by TNF relative to LPS could be due to a weaker ability of TNF to activate the
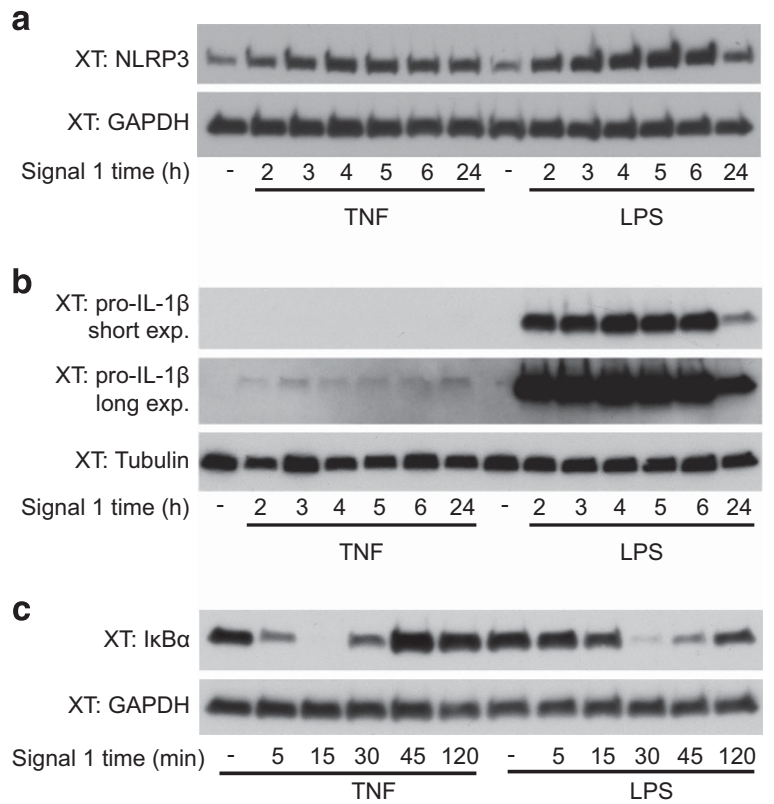

Figure 3 Relative to LPS, TNF induces weak and delayed expression of NLRP3 and pro-IL-1 $\beta$. (a, b) WT BMMs were left untreated ('-') or were stimulated with signal 1 (100 ng/ml LPS or $100 \mathrm{ng} / \mathrm{ml}$ TNF) over a $24 \mathrm{~h}$ time course. NLRP3 (a) and pro IL-1 $\beta$ (b) levels relative to GAPDH (a) or tubulin (b) (loading control) were measured by immunoblot. Data are representative of $n=3$ independent experiments. (c) WT BMMs were left untreated ('-') or were stimulated with signal $1(100 \mathrm{ng} / \mathrm{ml}$ LPS or $100 \mathrm{ng} / \mathrm{ml}$ TNF) over a $2 \mathrm{~h}$ time course. Degradation of $\mathrm{I}_{\kappa} \mathrm{B} \alpha$ relative to GAPDH (loading control) was measured by immunoblot. Data are representative of $n=4$ independent experiments. BMM, bone marrow-derived macrophages; IL, interleukin; LPS, lipopolysaccharide; TNF, tumor necrosis factor; WT, wild type.
NF- $\mathrm{BB}$ pathway in BMMs. To test this hypothesis, we examined the kinetics of I $\mathrm{KB} \alpha$ degradation (used as a proxy for NF-KB pathway activation) by microbial versus sterilepriming stimuli over a $2 \mathrm{~h}$ time course. Both LPS and TNF induced IкB $\alpha$ degradation and its resynthesis with similar intensity and kinetics (Figure 3c). This indicates that the weaker inflammasome responses generated by sterile signal 1 (TNF) were not due to an inability of this signal to fully activate the NF-KB pathway.

NLRP3 responses to sterile signals are also weaker in human macrophages

To determine whether the hierarchy of inflammasome responses to microbial and sterile signals that we observed in murine macrophages is conserved in humans, we examined NLRP3 activation by microbial (LPS) or sterile (TNF) signal 1 in combination with microbial (nigericin) or sterile (ATP) signal 2 in HMDMs (Figure 4). Because we found that 4 to $6 \mathrm{~h}$ priming rendered mouse BMMs responsive to both LPS and TNF (Figure 1 and data not shown), we primed HMDM for $4 \mathrm{~h}$ with LPS or TNF before activating NLRP3 with nigericin or ATP for $2 \mathrm{~h}$. In contrast to our observations in mouse macrophages, in HMDM NLRP3 induced very little cell death over control levels at this time, and ATP alone was cytotoxic (Figure 4a). However, the hierarchy of IL-1 $\beta$ release from HMDMs in response to microbial and sterile signal 1 and 2 (Figure 4b) mirrored that of mouse BMMs (Figure 1). In HMDMs, NLRP3-dependent IL-1 $\beta$ secretion in response to microbial signal 1 (LPS) and 2 (Nigericin) stimuli was far superior in magnitude to sterile signal 1 (TNF) and 2 (ATP) stimuli in any combination. Consistent with our observations in mouse BMMs, the combination of sterile signal 1 (TNF) and 2 (ATP) was the weakest inducer of IL- $1 \beta$ secretion (Figure $4 \mathrm{~b}$ ).

\section{DISCUSSION}

A key function of the immune system is to maintain the body's integrity. If we become infected our immune system fights the infection, and if we injure ourselves it repairs the wound. These two very different processes (defence versus repair) are surprisingly controlled by the same basic cellular machinery, for example, the NLRP3 inflammasome. Mechanisms by which NLRP3 may direct a response that is tailored to the circumstances of its activation are largely unknown. We recently reported one mechanism whereby in vivo responses may be tailored according to the NLRP3-activating signal; we found that neutrophils respond to only a subset of NLRP3 agonists, and NLRP3 activation in these cells drives IL- $1 \beta$ production but not IL-18 release. ${ }^{30}$ Thus, a subset of NLRP3 agonists may engender an IL-1 $\beta$-dominated response in vivo, through activating neutrophil NLRP3. In the present study, we examined whether the NLRP3 inflammasome drives distinct responses under conditions of microbial infection versus sterile injury using LPS+nigericin and TNF+ATP as model NLRP3-activating stimuli in macrophages. We revealed differences in both the kinetics and magnitude of NLRP3 responses 
a

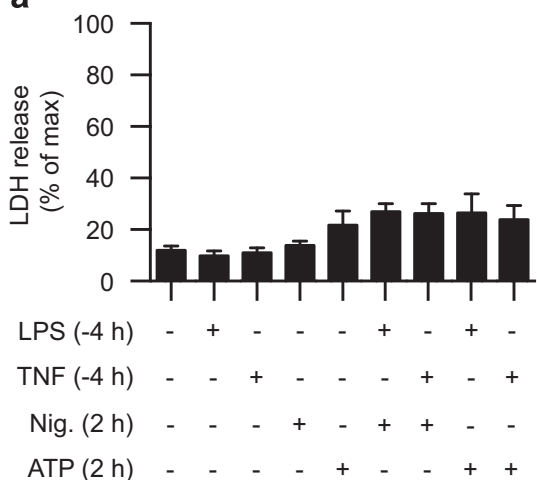

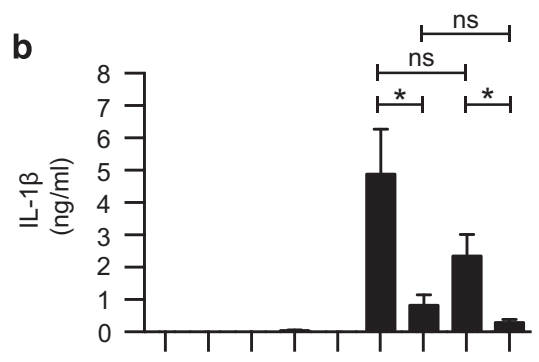

$\operatorname{LPS}(-4 \mathrm{~h})-+-\quad-++\quad+$

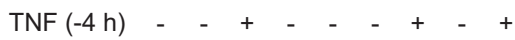

Nig. $(2 \mathrm{~h})-\mathrm{C}_{-}+\mathrm{C}^{-}+\mathrm{C}^{-}-$

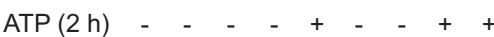

Figure 4 Sterile signals are weak activators of NLRP3 inflammasome responses in human macrophages. (a, b) HMDMs were left untreated ('-') or were stimulated with $100 \mathrm{ng} / \mathrm{ml}$ LPS or $100 \mathrm{ng} / \mathrm{ml}$ TNF for $4 \mathrm{~h}$ followed by $1.25 \mathrm{~mm}$ ATP or $5 \mu \mathrm{m}$ nigericin for $2 \mathrm{~h}$. Cell death was assessed by LDH assay (a), and ELISA quantified IL-1 $\beta$ release (b). Histograms are mean+s.e.m. of data pooled from $n=8$ experiments with cells prepared from independent donors. ELISA, enzyme-linked immunosorbent assay; HMDM, human monocyte-derived macrophage; IL, interleukin; LDH, lactate dehydrogenase; LPS, lipopolysaccharide; TNF, tumor necrosis factor.

generated by these stimuli in macrophages from mice and humans. We used LPS and TNF as prototypic microbial and sterile signal 1 stimuli, respectively, to prime the NLRP3 pathway in macrophages (for example, to induce the expression of the pathway sensor, NLRP3 and the caspase-1 substrate, pro-IL-1 $\beta$ ). We then used the bacterial toxin, nigericin, and ATP, an alarmin released from necrotic cells, as prototypic microbial and sterile signal 2 stimuli, respectively, to trigger the assembly of the NLRP3 inflammasome complex. We found that sterile signals generally produced weaker macrophage NLRP3 inflammasome responses relative to microbial signals. Specifically, NLRP3 inflammasome responses (caspase-1 cleavage, cell death and IL- $1 \beta$ secretion) were weakest when both signal 1 and signal 2 were sterile, and sterile signal 1 (TNF) primed for inflammasome responses with delayed kinetics relative to microbial signal 1 (LPS). Responses were somewhat faster and stronger when at least one of the two signals was microbe derived, and finally, the strongest and fastest response occurred when microbe-derived molecules were used for both signal 1 and signal 2. Together, these data suggest that microbial versus sterile signal 1 stimuli are distinct, both kinetically and in magnitude, in their ability to generate well-established inflammasome responses. This makes intuitive sense, as it should be a priority for the immune system to respond most rapidly and most robustly to the presence of infection to limit pathogen dissemination, even if such response comes at a cost to the surrounding tissue. Conversely, wound-healing responses are expected to be tempered and delayed, less urgent and indeed may only be appropriate when injury signals are present chronically. The differences in inflammasome responses to sterile versus microbial signals we observed (Figure 1) are broadly consistent with previous studies ${ }^{16,17}$ that first identified TNF as a sterile priming signal for the NLRP3 inflammasome.

The mechanisms underlying the tempered inflammasome response to sterile signal 1 are unknown. One potential explanation could be the different ability of microbial and sterile signal 1 to induce the inflammasome sensor NLRP3 (Figure 3). Because macrophages always express some NLRP3 protein, inflammasomes can be activated upon acute LPS priming through mechanisms independent of NLRP3 upregulation $^{31}$ (Figure 1), but increased expression of NLRP3 is largely responsible for the ability of LPS to prime over longer times. ${ }^{17}$ We found that NLRP3 expression was upregulated by signal 1 of both host and microbial origin. However, NLRP3 upregulation by TNF was weaker and more persistent than LPS-mediated NLRP3 upregulation (Figure 3). This might explain both the lower magnitude and longer duration of the inflammasome response to sterile (TNF) relative to microbial (LPS) signal 1. In addition, differences in signaling pathways triggered by LPS and TNF are likely to also have a role, particularly in acute priming. ${ }^{32,33}$ The reasons behind the different responses to sterile (ATP) versus microbial (nigericin) signal 2 are currently unclear because both are thought to activate the inflammasome pathway via common downstream mediator (that is, by inducing $\mathrm{K}+$ efflux from the cell ${ }^{5}$ ). One possibility is that heterogeneous expression of the P2X7R among macrophages supports NLRP3 signaling in only a subset of cells within the population.

Inflammasome-induced cell death was recently reported to depend on Gasdermin D cleavage by inflammatory caspases. ${ }^{3-36}$ In the canonical NLRP3 inflammasome pathway, Gasdermin D is processed by caspase-1. ${ }^{34-36}$ It is not known whether sterile and microbial signals differ in their ability to prime for, or induce, processing of Gasdermin D. However, as we have shown that such signals trigger distinct kinetics and magnitude of caspase-1 activation and pyroptosis, we would 
predict that the extent of Gasdermin D cleavage will also differ between sterile and microbial stimuli, but this remains to be tested. Although the hierarchy of NLRP3 responses to sterile versus microbial signals was conserved between human and mouse macrophages, interestingly, cell death responses in human macrophages appeared diminished relative to their murine counterparts. Whether this phenomenon reflects intra-species differences in the efficiency of caspase-1dependent Gasdermin D cleavage or pyroptosis execution is currently unknown.

Collectively, our data suggest that the responses elicited by the macrophage NLRP3 inflammasome pathway are distinct, depending on the nature of the activating signal (microbial product versus host-derived mediator that mimics sterile tissue injury). This is mechanistically unexpected, as responses to both stimuli are mediated by the same signaling complex (the NLRP3 inflammasome), but it makes biological sense, as these stimuli should trigger distinct types of immune responses (that is, antimicrobial versus tissue repair). Whether the quality of inflammasome-dependent responses to sterile damage is also different from the quality of responses generated to microbial infection is currently unknown. This will be fertile ground for future research elucidating inflammasome-dependent functional programs dedicated to resolving these two fundamentally different pathologies.

\section{CONFLICT OF INTEREST}

The authors declare no conflict of interest.

\section{ACKNOWLEDGEMENTS}

We thank members of the Schroder laboratory for helpful discussions on this project. This research was supported by a University of Queensland Early Career researcher grant (ECR102) to JSB and the Queensland Smart Futures Fund (fellowship to KS). KS is supported by an Australian Research Council Future Fellowship (FT130100361).

1 Medzhitov R. Origin and physiological roles of inflammation. Nature 2008; 454: 428-435.

2 Lamkanfi M, Dixit VM. Mechanisms and functions of inflammasomes. Cell 2014; 157: 1013-1022.

3 Schroder K, Tschopp J. The inflammasomes. Cell 2010; 140: 821-832.

4 Kapetanovic R, Bokil NJ, Sweet MJ. Innate immune perturbations, accumulating DAMPs and inflammasome dysregulation: a ticking time bomb in ageing. Ageing Res Rev 2015; 24: 40-53.

5 Munoz-Planillo R, Kuffa P, Martinez-Colon G, Smith BL, Rajendiran TM, Nunez G. K (+) efflux is the common trigger of NLRP3 inflammasome activation by bacterial toxins and particulate matter. Immunity 2013; 38: 1142-1153.

6 Gross O, Poeck H, Bscheider M, Dostert C, Hannesschlager N, Endres $\mathrm{S}$ et al. Syk kinase signalling couples to the NIrp3 inflammasome for anti-fungal host defence. Nature 2009; 459: 433-436.

7 Ketelut-Carneiro N, Silva GK, Rocha FA, Milanezi CM, Cavalcanti-Neto FF, Zamboni DS et al. IL-18 triggered by the NIrp3 inflammasome induces host innate resistance in a pulmonary model of fungal infection. $\mathrm{J}$ Immunol 2015; 194: 4507-4517.

8 Chen KW, Schroder K. Antimicrobial functions of inflammasomes. Curr Opin Microbiol 2013; 16: 311-318.
9 Broz P, Newton K, Lamkanfi M, Mariathasan S, Dixit VM, Monack DM. Redundant roles for inflammasome receptors NLRP3 and NLRC4 in host defense against Salmonella. J Exp Med 2010; 207: 1745-1755.

10 Ceballos-Olvera I, Sahoo M, Miller MA, Del Barrio L, Re F. Inflammasome-dependent pyroptosis and IL-18 protect against Burkholderia pseudomallei lung infection while IL-1beta is deleterious. PLoS Pathog 2011; 7: e1002452.

11 Broderick L, De Nardo D, Franklin BS, Hoffman HM, Latz E. The inflammasomes and autoinflammatory syndromes. Annu Rev Pathol 2015; 10: 395-424.

12 Martinon F, Petrilli V, Mayor A, Tardivel A, Tschopp J. Gout-associated uric acid crystals activate the NALP3 inflammasome. Nature 2006; 440: 237-241.

13 Masters SL, Dunne A, Subramanian SL, Hull RL, Tannahill GM, Sharp FA et al. Activation of the NLRP3 inflammasome by islet amyloid polypeptide provides a mechanism for enhanced IL-1beta in type 2 diabetes. Nat Immunol 2010; 11: 897-904.

14 Halle A, Hornung V, Petzold GC, Stewart CR, Monks BG, Reinheckel T et al. The NALP3 inflammasome is involved in the innate immune response to amyloid-beta. Nat Immunol 2008; 9: 857-865.

15 Heneka MT, Kummer MP, Stutz A, Delekate A, Schwartz S, Vieira-Saecker A et al. NLRP3 is activated in Alzheimer's disease and contributes to pathology in APP/PS1 mice. Nature 2013; 493: 674-678.

16 Franchi L, Eigenbrod T, Nunez G. Cutting edge: TNF-alpha mediates sensitization to ATP and silica via the NLRP3 inflammasome in the absence of microbial stimulation. J Immunol 2009; 183: 792-796.

17 Bauernfeind FG, Horvath G, Stutz A, Alnemri ES, MacDonald K, Speert D et al. Cutting edge: NF-kappaB activating pattern recognition and cytokine receptors license NLRP3 inflammasome activation by regulating NLRP3 expression. J Immunol 2009; 183: 787-791.

18 Sutterwala FS, Haasken S, Cassel SL. Mechanism of NLRP3 inflammasome activation. Ann N Y Acad Sci 2014; 1319: 82-95.

19 Vande Walle L, Van Opdenbosch N, Jacques P, Fossoul A, Verheugen $E$, Vogel $P$ et al. Negative regulation of the NLRP3 inflammasome by A20 protects against arthritis. Nature 2014; 512: 69-73.

20 Duong $\mathrm{BH}$, Onizawa M, Oses-Prieto JA, Advincula R, Burlingame A, Malynn BA et al. A20 restricts ubiquitination of pro-interleukin-1beta protein complexes and suppresses NLRP3 inflammasome activity. Immunity 2015; 42: 55-67.

21 Hise AG, Tomalka J, Ganesan S, Patel K, Hall BA, Brown GD et al. An essential role for the NLRP3 inflammasome in host defense against the human fungal pathogen Candida albicans. Cell Host Microbe 2009; 5: 487-497.

22 Schweneker K, Gorka O, Schweneker M, Poeck H, Tschopp J, Peschel $\mathrm{C}$ et al. The mycobacterial cord factor adjuvant analogue trehalose-6,6'-dibehenate (TDB) activates the NIrp3 inflammasome. Immunobiology 2013; 218: 664-673.

23 Dinarello CA, Cannon JG, Wolff SM, Bernheim HA, Beutler B, Cerami A et al. Tumor necrosis factor (cachectin) is an endogenous pyrogen and induces production of interleukin 1. J Exp Med 1986; 163: 1433-1450.

24 Chow MT, Duret H, Andrews DM, Faveeuw C, Moller A, Smyth MJ et al. Type I NKT-cell-mediated TNF-alpha is a positive regulator of NLRP3 inflammasome priming. Eur J Immunol 2014; 44: 2111-2120.

25 Feldmann M. Development of anti-TNF therapy for rheumatoid arthritis. Nat Rev Immunol 2002; 2: 364-371.

26 Uysal KT, Wiesbrock SM, Marino MW, Hotamisligil GS. Protection from obesity-induced insulin resistance in mice lacking TNF-alpha function. Nature 1997; 389: 610-614.

27 Kuida K, Lippke JA, Ku G, Harding MW, Livingston DJ, Su MS et al. Altered cytokine export and apoptosis in mice deficient in interleukin-1 beta converting enzyme. Science 1995; 267: 2000-2003.

28 Schroder K, Irvine KM, Taylor MS, Bokil NJ, Le Cao KA, Masterman KA et al. Conservation and divergence in Toll-like receptor 4-regulated gene expression in primary human versus mouse macrophages. Proc Natl Acad Sci USA 2012; 109: E944-E953.

29 Guarda G, Braun M, Staehli F, Tardivel A, Mattmann C, Forster I et al. Type I interferon inhibits interleukin-1 production and inflammasome activation. Immunity 2011; 34: 213-223.

30 Chen KW, Bezbradica JS, Groß CJ, Wall AA, Sweet MJ, Stow JL et al. The murine neutrophil NLRP3 inflammasome is activated by soluble but not particulate or crystalline agonists. Eur 
J Immunol 2016; e-pub ahead of print 1 February 2016; doi:10.1002/eji.201545943.

31 Schroder K, Sagulenko V, Zamoshnikova A, Richards AA, Cridland JA, Irvine $\mathrm{KM}$ et al. Acute lipopolysaccharide priming boosts inflammasome activation independently of inflammasome sensor induction. Immunobiology 2012; 217: 1325-1329.

32 Lin KM, Hu W, Troutman TD, Jennings M, Brewer T, Li X et al. IRAK-1 bypasses priming and directly links TLRs to rapid NLRP3 inflammasome activation. Proc Natl Acad Sci USA 2014; 111: 775-780.

33 Fernandes-Alnemri T, Kang S, Anderson C, Sagara J, Fitzgerald KA, Alnemri ES. Cutting edge: TLR signaling licenses IRAK1 for rapid activation of the NLRP3 inflammasome. J Immunol 2013; 191: 3995-3999.

34 He WT, Wan H, Hu L, Chen P, Wang X, Huang Z et al. Gasdermin D is an executor of pyroptosis and required for interleukin-1beta secretion. Cell Res 2015; 25: 1285-1298.

35 Shi J, Zhao Y, Wang K, Shi X, Wang Y, Huang $\mathrm{H}$ et al. Cleavage of GSDMD by inflammatory caspases determines pyroptotic cell death. Nature 2015; 526: 660-665.
36 Kayagaki N, Stowe IB, Lee BL, O'Rourke K, Anderson K, Warming S et al. Caspase-11 cleaves gasdermin $D$ for non-canonical inflammasome signalling. Nature 2015; 526: 666-671.

(c) (1) $(\Theta)$ This work is licensed under a Creative Commons Attribution-NonCommercial-NoDerivs 4.0 International License. The images or other third party material in this article are included in the article's Creative Commons license, unless indicated otherwise in the credit line; if the material is not included under the Creative Commons license, users will need to obtain permission from the license holder to reproduce the material. To view a copy of this license, visit http:// creativecommons.org/licenses/by-nc-nd/4.0/ 